\title{
Comparative investigation of different silane surface functionalizations of fullerene-like $\mathrm{WS}_{2}$
}

\author{
Dietmar Haba $^{1}(\mathbb{D}) \cdot$ Thomas Griesser $^{2}$ - Ulrich Müller ${ }^{1}$ • \\ Andreas J. Brunner ${ }^{1}$
}

Received: 13 February 2015/Accepted: 17 April 2015/Published online: 6 May 2015

(C) Springer Science+Business Media New York 2015

\begin{abstract}
Inorganic fullerene-like tungsten disulfide (IF$\mathrm{WS}_{2}$ ) nanoparticles are useful additives for polymers and lubricating agents, in particular when their surfaces are functionalized by silane modifiers. However, both the success of such a silanization reaction and its effect on the final dispersion quality are still doubtful. In this work, IF-WS ${ }_{2}$ are functionalized using three different silane modifiers and investigated with $\mathrm{X}$-ray photoelectron spectrometry, infrared spectroscopy, titration, thermogravimetric analysis, and mass spectroscopy. Eventually, they are dispersed within ethanol by sonication to compare the dispersing behavior. The combination of the different analytical techniques revealed that the IF-WS 2 surfaces can be functionalized with two of the used silane modifiers, while the third one was repeatedly unsuccessful. The amount of Si on the particles seems to be a fairly clear indication for the success of the functionalization reaction. The IF-WS ${ }_{2}$ seems to oxidize during the functionalization process, probably producing acidic $\mathrm{SO}_{2}$ or $\mathrm{SO}_{3}$, which can fully acidify a basic surface modifier. The executed treatment without any added silane modifier improved the dispersibility of the IF-WS ${ }_{2}$ within ethanol to some extent, but added modifiers deteriorated it significantly. TEM images indicate that IF-WS 2 particles form aggregates, which might be the reason for the limited dispersibility.
\end{abstract}

Electronic supplementary material The online version of this article (doi:10.1007/s10853-015-9039-4) contains supplementary material, which is available to authorized users.

Dietmar Haba

Dietmar.Haba@empa.ch

1 Empa - Swiss Federal Laboratories for Materials Science and Technology, Dübendorf, Switzerland

2 Montanuniversität Leoben, Leoben, Austria

\section{Introduction}

Inorganic fullerene-like tungsten disulfide (IF-WS $\left.{ }_{2}\right)$ nanoparticles are a relatively new class of nanomaterials. They are best known for their outstanding lubricating effect $[1,2]$, but there are also promising reports on their use as a nanofiller for various polymers [3]. While most research in this field was done on thermoplastics, a few publications dealt with epoxy-IF- $\mathrm{WS}_{2}$ nanocomposites and showed substantial improvements in adhesion fracture toughness and other mechanical properties $[4,5]$.

Despite the rather inert nature of IF-WS ${ }_{2}$ nanoparticles, it was indicated that it is possible to graft silane surface modifiers onto them in order to improve their dispersibility within lubricating oils [2] and epoxy resins [5], respectively. The lubricating and toughening effects of silanefunctionalized IF-WS $\mathrm{W}_{2}$ were superior to those of unfunctionalized IF-WS $\mathrm{W}_{2}$ While the exact course of this silanization reaction is not known, it was assumed that $\mathrm{H}_{2} \mathrm{O}$ located at defect sites of the IF-WS ${ }_{2}$ plays an important role. Besides the reported improvements, however, a few X-ray photoelectron spectroscopy (XPS) and infrared (IR) spectroscopy measurements are the only indications that the silanization reaction did take place $[2,5]$.

In this work, we investigate the feasibility of a surface functionalization of IF- $\mathrm{WS}_{2}$ with selected silane modifiers for later use as a nanofiller in epoxy nanocomposites. The aim of the functionalization was to improve the dispersibility of the IF-WS $\mathrm{Ws}_{2}$ as well as their bonding to an epoxy matrix. Special emphasis was given to detailed characterization of the obtained powders with various analytical methods.

Chlorosilanes are known to be much more reactive than alkoxysilanes, which is not only beneficial for the modification process, but also limits the available functional groups, as many would react with the chlorosilane 
themselves. Alkoxysilanes, in contrast, are available with various functional end groups and are usually much more easy to handle which is an important requirement for a possible industrial application.

In this work, treated and untreated IF-WS ${ }_{2}$ are dispersed within ethanol by sonication for different time periods. The aim was not to obtain optimum dispersion quality but rather to investigate the effect of the surface functionalization on it. Sonication was chosen as it is a rather welldefined process and as it is a frequently reported technique for dispersing IF-WS $\mathrm{WS}_{2}$ in organic liquids [2, 3, 5-8]. A later publication will deal with the dispersion of these particles within epoxy and the resulting properties.

\section{Experimental}

\section{Materials}

The black IF-WS ${ }_{2}$ powder with the trade name Nanolub $R$ was received from NanoMaterials Ltd. (Israel); this source has been used in earlier research published by other groups $[2,4,5,9]$. According to the manufacturer, it was produced by high-temperature solid-gas synthesis, the primary particles had diameters of 40-300 nm and they consisted of $10-100 \mathrm{WS}_{2}$ layers, with an interlayer spacing of $0.62 \mathrm{~nm}[10]$.

The silane surface modifiers are depicted in Fig. 1. The chosen chorosilane was hexyltrichlorosilane (HTCS, $85 \%$ purity); its rather short carbon chain is expected to interact well with a fairly polar epoxy resin. Moreover, it does not act as a plasticizer as strongly as long carbon chains do. The chosen alkoxysilane modifiers were 3-glycidoxypropyltrimethoxysilane (GTMS, $98 \%$ purity) and 3-(2-aminoethylamino)propyltrimethoxysilane (AATMS, $80 \%$ purity). Both are expected to improve the miscibility of nanoparticles with epoxy resin and their functional groups should react with either epoxy or the amine hardener, promising good bonding between the nanoparticles and the epoxy resin. All silane modifiers were obtained from Sigma-Aldrich (USA).

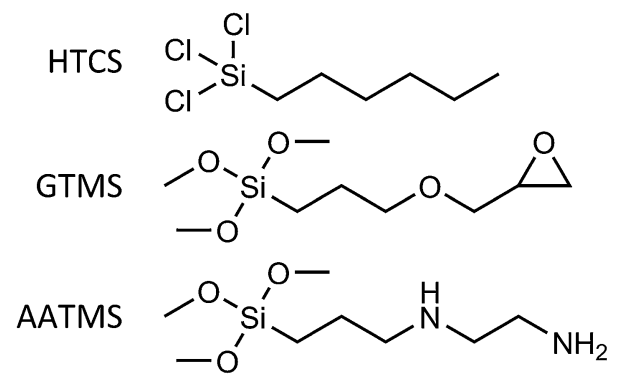

Fig. 1 Used silane surface modifiers

\section{Processing}

For the surface functionalization with the chlorosilane HTCS, chloroform $\left(\mathrm{CHCl}_{3}\right)$ was used as a solvent (stabilized with $1 \%$ ethanol, $99 \%$ purity), while for the functionalization with the alkoxysilanes GTMS and AATMS, ethanol $(\mathrm{EtOH})$ was used (denaturated with $5 \%$ isopropyl alcohol, $99.9 \%$ purity). Approx. $100 \mathrm{ml}$ of these solvents were added to $1.00 \mathrm{~g} \mathrm{IF-WS}$ and sonicated for $1 \mathrm{~min}$ with a $200 \mathrm{~W}$ ultrasonic wave generator (Bandelin Sonoplus HD 2200 ) oscillating a $\mathrm{Ti}_{6} \mathrm{Al}_{4} \mathrm{~V}$ sonotronde $(\varnothing 3 \mathrm{~mm})$ at maximum intensity. Within a few seconds, the temperature rose to the boiling point of the respective solvent. In the case of the $\mathrm{CHCl}_{3}$ suspension, all subsequent steps were done under exclusion of air as the highly reactive chlorosilane might otherwise react with air moisture.

The suspensions were further sonicated in an ultrasonic bath at the boiling temperature of the respective solvent while being stirred with a PTFE-mantled overhead stirrer. In separate flasks, $100 \mathrm{mg}$ of the respective surface modifier were dissolved within the respective solvents; these solutions were then added dropwise to the suspensions with a syringe. After $60 \mathrm{~min}$, the sonication was stopped and the suspensions were further stirred with magnetic stirrers. The $\mathrm{EtOH}$ suspensions were stirred overnight at $90{ }^{\circ} \mathrm{C}$ under reflux while the $\mathrm{CHCl}_{3}$ suspensions were stirred closed at room temperature.

The day after, the particles were separated from the solvent by centrifuging at $2000 \mathrm{~g}$ for $3 \mathrm{~min}$ per pass. The liquid phase was decanted and kept for further analysis. When all the solvent was removed, fresh solvent was added, the suspensions were mixed thoroughly, and then centrifuged again. This was done three times in order to properly remove the remaining unreacted modifier as well as soluble reaction products. When the liquid phase showed still a gray color tone after centrifuging, the centrifuging step was redone at higher speed and longer times, up to $4000 \mathrm{~g}$ for $30 \mathrm{~min}$. The remaining slurries were heated to $50{ }^{\circ} \mathrm{C}$ in vacuum for $1 \mathrm{~h}$ to remove the remaining solvent. The powder was then ground with a mortar and a pestle. Finally, the powders were tempered at $100{ }^{\circ} \mathrm{C}$ in vacuum for $1 \mathrm{~h}$ to make the residual unreacted silane groups react.

These processes were repeated both with three times the amount of surface modifier and without surface modifier. Table 1 gives an overview over all treated IF-WS 2 powders. All powders were stored in a desiccator over calcium chloride in vacuum (at approx. 5 mbar).

Suspensions of treated and untreated IF-WS ${ }_{2}$ within $\mathrm{EtOH}$ were sonicated in order to determine which average agglomerate size can be obtained that way. For that purpose, $50 \mathrm{ml}$ of EtOH were added to $18 \mathrm{mg} \mathrm{IF-WS}$ in a roundbottom flask (particle volume fraction $\varphi_{\mathrm{p}} \approx 5 \times 10^{-5}$ ). The suspensions were sonicated the same way as mentioned above for up to $60 \mathrm{~min}$. 
Table 1 Overview over all treated IF-WS ${ }_{2}$ powders

\begin{tabular}{llll}
\hline Denomination & \multicolumn{2}{l}{ Surface modifier } & Solvent \\
\cline { 2 - 3 } & Type & Mass/mg & \\
\hline Ref-EtOH & & & EtOH \\
Ref-CHCl & & $\mathrm{CHCl}_{3}$ \\
GTMS-1 & GTMS & 100 & $\mathrm{EtOH}$ \\
GTMS-3 & GTMS & 300 & $\mathrm{EtOH}$ \\
AATMS-1 & AATMS & 100 & $\mathrm{EtOH}$ \\
AATMS-3 & AATMS & 300 & $\mathrm{EtOH}$ \\
HTCS-1 & HTCS & 100 & $\mathrm{CHCl}_{3}$ \\
HTCS 3 & HTCS & 300 & $\mathrm{CHCl}_{3}$ \\
\hline
\end{tabular}

\section{Characterization}

Transmission IR specta were obtained from 4000 to $400 \mathrm{~cm}^{-1}$ with $1.9 \mathrm{~cm}^{-1}$ step size (64 scans, Bruker Tensor 27). Approx. $0.5 \mathrm{mg}$ of IF-WS 2 powders were ground in a mortar together with $150 \mathrm{mg} \mathrm{KBr}$ (trade name Spectranal, delivered by Fluka) and subsequently compressed at $0.75 \mathrm{GPa}$ for $2 \mathrm{~min}$.

All treated IF-WS 2 powders were investigated using an XPS spectrometer (Physical Electronics Quantum 2000) to gather additional information on the success of the silanization reaction. The machine uses monochromatic Al K $\alpha$ X-rays $(h v=1486.7 \mathrm{eV})$ and operates at a pressure of $3 \times 10^{-7} \mathrm{~Pa}$ at room temperature. The electron take-off angle was $45^{\circ}$ and the analyzer was operated in the constant pass energy mode at $58.7 \mathrm{eV}$. The beam diameter chosen was around $150 \mu \mathrm{m}$, and spectra were recorded with a step size of $0.25 \mathrm{eV}$. The powders were pressed into an In foil and kept in vacuum at least $16 \mathrm{~h}$ before the measurement. Electron and $\mathrm{Ar}^{+}$neutralizers were used to compensate for possible surface charging. The samples were measured in randomized order. The spectra were analyzed using the software MultiPak 8.2B. A Shirley background subtraction was done on every peak, before its area was calculated by numerical integration. As the $\mathrm{W}_{4 f}$ peaks overlap with a $\mathrm{W}_{5 p 3 / 2}$ peak, their areas were instead calculated by fitting those curves with a Gaussian-Lorentzian function, and only the area belonging to the $\mathrm{W}_{4 f}$ peaks were used. The atomic fractions of the individual elements were calculated from these peak areas using the corrected relative sensitivity factors calculated by the software.

As one of the chosen silane surface modifiers, AATMS contains two basic amine groups, its content is measurable by acid-base titration. This was done on AATMS, on the AATMS-treated IF-WS $\mathrm{W}_{2}$ powders and on the EtOH rest from the two AATMS modifications (called AATMS$X$-rest-EtOH). For the titration, $100 \mathrm{mg}$ of AATMS were dissolved in $25 \mathrm{ml}$ of $\mathrm{EtOH}$ and another $25 \mathrm{ml}$ of $\mathrm{H}_{2} \mathrm{O}$ were added; $100 \mathrm{mg}$ of the AATMS-treated IF-WS 2 powders were dispersed in $25 \mathrm{ml}$ of $\mathrm{H}_{2} \mathrm{O}$; and $25 \mathrm{ml}$ of the AATMS-X-rest-EtOH were mixed with $25 \mathrm{ml}$ of $\mathrm{H}_{2} \mathrm{O}$. The titration was done with $10 \mathrm{mmol} / \mathrm{l} \mathrm{HCl}$ or $\mathrm{NaOH}$ in $\mathrm{H}_{2} \mathrm{O}$ using a calibrated $\mathrm{pH}$ probe. This $\mathrm{H}_{2} \mathrm{O}$ had been freshly purified to Grade 1 (Thermo Scientific Barnstead NanoPure) [11]. The titration curves were evaluated using the CurTiPot software [12].

Thermogravimetric analysis (TGA) was performed in order to determine how effective the silanization had worked (Perkin Elmer TGA 7). Before the measurement, the powders were heated to $50{ }^{\circ} \mathrm{C}$ in vacuum for several hours in order to assure that they were dry. Roughly $6 \mathrm{mg}$ of treated IF-WS 2 powders were heated with $20^{\circ} \mathrm{C} / \mathrm{min}$ under He flow (purity 4.6). At $600{ }^{\circ} \mathrm{C}$, the gas was switched to $\mathrm{O}_{2}$ (purity 2.5 ).

In order to get more information on the origin of the mass loss in the TGA, $100 \mathrm{mg}$ of HTCS-3 and AATMS-3 were further investigated by TGA combined with mass spectrometry (TG/MS, Netzsch STA 409 CD with QMS 403 $C$ ) at $20^{\circ} \mathrm{C} / \mathrm{min}$ under He flow (purity 4.6).

Scanning electron microscopy (SEM) images were taken to determine the agglomerate sizes of treated and untreated powders after various sonication times. For this purpose, EtOH suspensions were applied dropwise to a $\mathrm{Si}$ wafer and dried at room temperature as it had been done earlier [2]. Images were gathered with a FEI NovaNanoSEM 230 with a Schottky field emission electron source operating at $5 \mathrm{kV}$, imaging secondary electrons with a through-the-lens detector at $3 \times 10^{-7}$ Pa pressure.

Dynamic light scattering (DLS) measurements were performed at $25.0{ }^{\circ} \mathrm{C}$ (Malvern Zetasizer Nano Z, laser wavelength $534 \mathrm{~nm}$ ). While this technique is best suited for the measurement of the diameters of well-dispersed spherical primary particles with sizes of 1 to $1000 \mathrm{~nm}$ [13], it has also been successfully used for quantifying agglomerate sizes [14-18]. EtOH suspensions were diluted with additional EtOH 1:10 (resulting particle volume fraction $\varphi_{\mathrm{p}} \approx 5 \times 10^{-} 6$ ). The viscosity of neat EtOH was used for the calculation of particle diameters. Presented values are the means of 15 individual measurements of $30 \mathrm{~s}$ each. As recommended by the respective ISO standard [19], the intensity-weighted average particle diameter $\bar{x}_{\text {DLS }}$ was used for evaluation.

For transmission electron microscopy (TEM), a drop of EtOH-IF-WS $\mathrm{W}_{2}$ suspension (sonicated for $60 \mathrm{~min}$ ) was placed on a TEM grid and allowed to dry. The images were gathered in a Schottky field emission TEM (JEOL 2200FS TEM/STEM) operating at $200 \mathrm{kV}$. 


\section{Results and discussion}

As mentioned above, there are two reports indicating that IF-WS 2 can be functionalized with chlorosilane or alkoxysilane surface modifiers $[2,5]$. As the underlying chemical reaction is, however, not fully understood so far, particular emphasis was given to a critical characterization with different methods.

\section{IR spectroscopy}

According to literature reports, the presence of surface modifier on IF-WS 2 can be detected by transmission IR spectroscopy, where IR bands at 2962, 2922 and $2852 \mathrm{~cm}^{-1}$ were attributed to - $\mathrm{CH} 3$ and - $\mathrm{CH} 2-$ groups [2]. Indeed, all treated IF-WS 2 showed IR bands at exactly these positions, even Ref-EtOH, which has no surface modifier on it; moreover, these bands were also observed in fresh $\mathrm{KBr}$ (data given in the Online Resource). Thus, these IR signals originate most likely from small impurities in the $\mathrm{KBr}$ powder rather than from an organic modifier. There was no significant increase observed from the fresh $\mathrm{KBr}$ to any of the samples of treated IF-WS ${ }_{2}$ This might be due to the high absorptivity of IF-WS $\mathrm{W}_{2}$ : Possible IR signals from the functionalization might have been reduced to below the noise level.

\section{XPS}

XPS survey scans showed that the elements present on the surfaces of the treated IF-WS ${ }_{2}$ powders were mostly those expected: $\mathrm{W}, \mathrm{S}, \mathrm{C}, \mathrm{O}, \mathrm{Si}$, and $\mathrm{N}$. Besides these, there was a small signal of In, which stems from the used foil, and a small signal of $\mathrm{Se}$, which is in traces often present in transition metal dichalcogenides. No signal of residual $\mathrm{Cl}$ was detected around $200 \mathrm{eV}$, indicating that no unreacted HTCS was left.

The highly resolved XPS scans are given in Fig. 2. The $\mathrm{W}_{4 f}$ peaks did not change for any sample, indicating that the surface functionalization did not alter the chemical state of the $\mathrm{W}$. A $\mathrm{WO}_{x}$ signal might be seen as a shoulder around $36.0 \mathrm{eV}$ as was stated earlier [2,5], but it is too small to draw a clear conclusion from.

The shape of the $S_{2 p}$ peaks did not change, but a side peak formed at higher binding energy in some cases. Späth et al. have already reported such a side peak in untreated, but aged IF-WS $\mathrm{WS}_{2}$ and attributed it to $\mathrm{SO}_{x}$ [9], and this interpretation is shared in the present work. As it was assumed that this side peak came from $S_{2 p}$ as well, its area was composed in the measurement of the $\mathrm{S}$ content.

Both $\mathrm{O}_{1 s}$ and $\mathrm{C}_{1 s}$ are compounded of at least two different peaks each, the ratio of which varies among the samples. The binding energy of $\mathrm{Si}_{2 p}$ of approx. $102.4 \mathrm{eV}$ is in good agreement with that reported for siloxanes [20]. A broad, low-intensity peak appeared to be present for all samples overlapping with the $\mathrm{Si}_{2 p}$ peak (see Fig. 2); as this peak was assumed to be a signal from the $\mathrm{W}$, it was removed by background subtraction before calculation of the $\mathrm{Si}_{2 p}$ peak area.

The measured atomic fractions of the individual elements are given in Table 2. Note that the absolute measurement uncertainty of atomic fractions determined by XPS is usually in the range of $\pm 10 \%$, while the relative differences between the individual samples might be measured more accurately. The detection limit depends on the investigated signal and on the background and lies usually at atomic fractions below $1.0 \%$.

The S/W ratio was generally slightly lower than 2 . This might be explained with the substitution of some $\mathrm{S}$ atoms by Se, or by the fact that some IF-WS ${ }_{2}$ nanoparticles might be broken up and $\mathrm{WO}_{3}$ from their core might have been measured. Given the limited accuracy of XPS with respect to quantification of composition, however, no further conclusions can be drawn from that. Even though the measurement order was random, the high correlation between similarly treated powders suggests that the $\mathrm{S} / \mathrm{W}$ ratio is indeed affected by the used modifier and/or the used solvent.

The increase in $\mathrm{C}_{1 s}$ content might be used as an indication for a successful functionalization as it has been done earlier $[2,5]$. However, while the $\mathrm{C}$ content is very low in Ref-EtOH, it is much higher in $\mathrm{Ref}-\mathrm{CHCl}_{3}$; this shows that an increase in the $\mathrm{C}_{1 s}$ peak alone does not necessarily provide proof of successful functionalization. Due to the high surface sensitivity of XPS, already small amounts of contaminants affect the measurement considerably, and C is known to be a ubiquitous contaminant. The shift in the $\mathrm{C}_{1 s}$ peak of Ref- $\mathrm{CHCl}_{3}$ is not significantly different from that of the functionalized powders neither.

The same is true for the $\mathrm{O}_{1 s}$ peak: while it increases strongly and shifts after functionalization, an increase or shift alone is not sufficient to tell whether the functionalization was successful. The strong $\mathrm{N}_{1 s}$ peak of the AATMS-functionalized powders agrees well with the assumed presence of amines and thus indicates that those powders have been functionalized successfully. However, Ref- $\mathrm{CHCl}_{3}$ and some powders functionalized with $\mathrm{N}$-free modifiers showed clear $\mathrm{N}_{1 s}$ peaks as well, which is again presumably due to contaminants.

In contrast, the $\mathrm{Si}_{2 p}$ peak is clearly measurable for some samples and not detectable for others. It hence seems that this peak provides clear evidence on whether the functionalization process was successful. It can therefore be concluded that both functionalization reactions with GTMS 

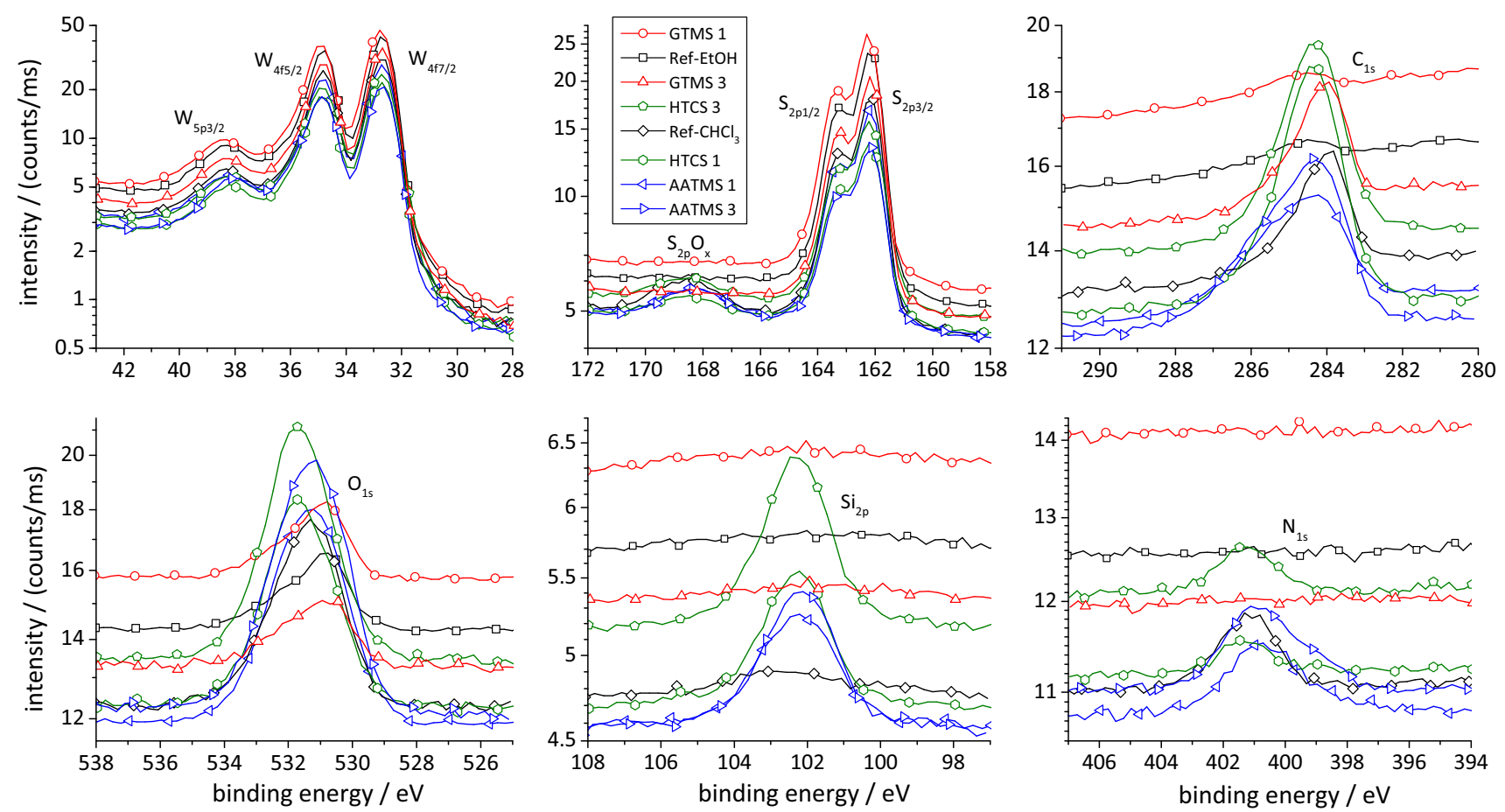

Fig. 2 XPS spectra of all treated IF-WS 2 powders with the presumed assignment

Table 2 Atomic fractions of the investigated elements as calculated from the curves in Fig. 2 and ratios thereof

\begin{tabular}{|c|c|c|c|c|c|c|c|c|}
\hline & $\begin{array}{l}\text { W } \\
\text { at.\% }\end{array}$ & $\begin{array}{l}\mathrm{S} \\
\text { at.\% }\end{array}$ & $\begin{array}{l}\mathrm{C} \\
\text { at.\% }\end{array}$ & $\begin{array}{l}\mathrm{O} \\
\text { at. } \%\end{array}$ & $\begin{array}{l}\mathrm{Si} \\
\text { at.\% }\end{array}$ & $\begin{array}{l}\mathrm{N} \\
\text { at.\% }\end{array}$ & S/W & $\begin{array}{l}\mathrm{SO}_{x} / \mathrm{S} \\
\%\end{array}$ \\
\hline Ref-EtOH & 31.4 & 55.2 & 3.0 & 10.4 & $*$ & $*$ & 1.76 & $*$ \\
\hline Ref- $\mathrm{CHCl}_{3}$ & 19.1 & 37.4 & 17.4 & 21.2 & $*$ & 4.4 & 1.96 & 8.9 \\
\hline GTMS-1 & 30.8 & 54.5 & 4.7 & 9.3 & $*$ & $*$ & 1.77 & $*$ \\
\hline GTMS-3 & 25.2 & 44.1 & 21.9 & 8.4 & $*$ & $*$ & 1.75 & $*$ \\
\hline AATMS-1 & 15.8 & 30.8 & 22.6 & 23.4 & 3.4 & 4.0 & 1.94 & 7.1 \\
\hline AATMS-3 & 11.3 & 21.5 & 31.7 & 26.1 & 4.2 & 5.2 & 1.90 & 11.1 \\
\hline HTCS-1 & 12.3 & 22.9 & 38.3 & 20.7 & 4.3 & 1.5 & 1.86 & 6.2 \\
\hline HTCS 3 & 13.3 & 24.7 & 31.1 & 23.0 & 5.6 & 2.3 & 1.85 & 6.5 \\
\hline
\end{tabular}

* Signal below the detection limit

were unsuccessful, while those with AATMS and those with HTCS were all successful. It is unclear why the functionalization with AATMS should be successful when that with GTMS is not as the type of reaction is the same, but the increase in the $\mathrm{C}_{1 s}, \mathrm{O}_{1 s}$ and $\mathrm{N}_{1 s}$ peaks support this interpretation. Moreover, it is not clear whether the silane modifiers attach to the IF-WS 2 by chemical bonds or just by physisorption.

The presence of the $\mathrm{SO}_{x}$ peak correlates strongly with the $\mathrm{O}$ content, indicating that it is indeed due to oxidized $\mathrm{S}$. While Späth et al. showed that IF-WS $\mathrm{W}_{2}$ oxidize upon exposure to ambient air for long time periods (3 years) [9], we can see here that the performed treatment can cause oxidation as well; the $\mathrm{SO}_{x}$ peak of Ref-CHCl${ }_{3}$ shows that the presence of silanes was not necessary for an oxidation reaction. Rather, the reason for this oxidation might lie in the short sonication at the beginning of the treatment. The high local temperatures during the sonication of up to $5000 \mathrm{~K}[21]$ might lead to the degradation of the IF-WS or the used solvents and the resulting formation of oxidative degradation products. Späth et al. assumed that the oxidized $\mathrm{S}$ might be present in the form of $\mathrm{SO}_{4}^{2-}$. In order to test that, small amounts of treated IF-WS $\mathrm{W}_{2}$ powders and of EtOH rests left from the treatment were mixed with $\mathrm{H}_{2} \mathrm{O}$ and $\mathrm{BaCl}_{2}$. As no precipitate formed in any of these cases, the presence of $\mathrm{SO}_{4}^{2-}$ seems unlikely.

Due to the contaminations with $\mathrm{C}, \mathrm{O}$, and $\mathrm{N}$, only limited conclusions can be drawn from the atomic ratios of these. Analyzing the amount of shifting of the $\mathrm{C}_{1 s}$ and the $\mathrm{O}_{1 s}$ peaks did not yield conclusive facts neither.

\section{Titration}

The well-reproducible titration curve of AATMS shows two $\mathrm{pH}$ steps as it is typical for diprotic bases. However, significantly more than double the amount of $\mathrm{HCl}$ had to be added until the second $\mathrm{pH}$ step than until the first one, namely 2.4 times that much (see Fig. 3). This indicates that some portion of the AATMS molecules, namely $34 \%$, had been monoprotonated already before their delivery, making one of their amine groups an ammonium group. This is not 
unexpected, given the limited purity of the AATMS and the fact that amines are easily protonated.

The amine group content in AATMS can be quantified by subtracting the amount of $\mathrm{HCl}$ necessary until the first $\mathrm{pH}$ step (where half of the molecules are protonated once) from that necessary until the second $\mathrm{pH}$ step (where half of the molecules are protonated twice). More precise results were obtained with the titration curve fitting software. The measured acid dissociation constants $K_{\mathrm{a}}$ calculated by curve fitting were $10^{-6.57}$ and $10^{-9.77}$ and therefore similar to $10^{-6.86}$ and $10^{-9.92}$ which are stated for ethylenediamine [22]. The amine group content was as high as $9.5 \mathrm{mmol} / \mathrm{g}$ and hence higher than the theoretically expected value of $9.0 \mathrm{mmol} / \mathrm{g}$. It seems, therefore, that some diamine was present in the form of the AATMS's precursor, that is to say, without a trimethoxysilane group. Once again, this is not unexpected given AATMS's limited purity. All subsequent calculations were done assuming an amine group content of $9.5 \mathrm{mmol} / \mathrm{g}$.

A comparable titration was done on AATMS-3-restEtOH. The titration curve was again well reproducible, and once again showed two $\mathrm{pH}$ steps at the same $\mathrm{pH}$ values, indicating that there was still AATMS left in the EtOH after centrifugation. This time, the results indicate that $92 \%$ of the AATMS molecules have been protonated already once, which is significantly more than before the treatment. Thus, acidic groups have been added or created during the treatment. Considering the XPS results, this is most likely due to the $\mathrm{SO}_{x}$ surface groups that were sometimes produced during the treatment. It is worth noting that untreated IF-WS $\mathrm{W}_{2}$ leads to acidic $\mathrm{pH}$ when dispersed within $\mathrm{H}_{2} \mathrm{O}$ which may stem for the $\mathrm{H}_{2} \mathrm{~S}$-rich atmosphere usually used for their synthesis [23, 24],

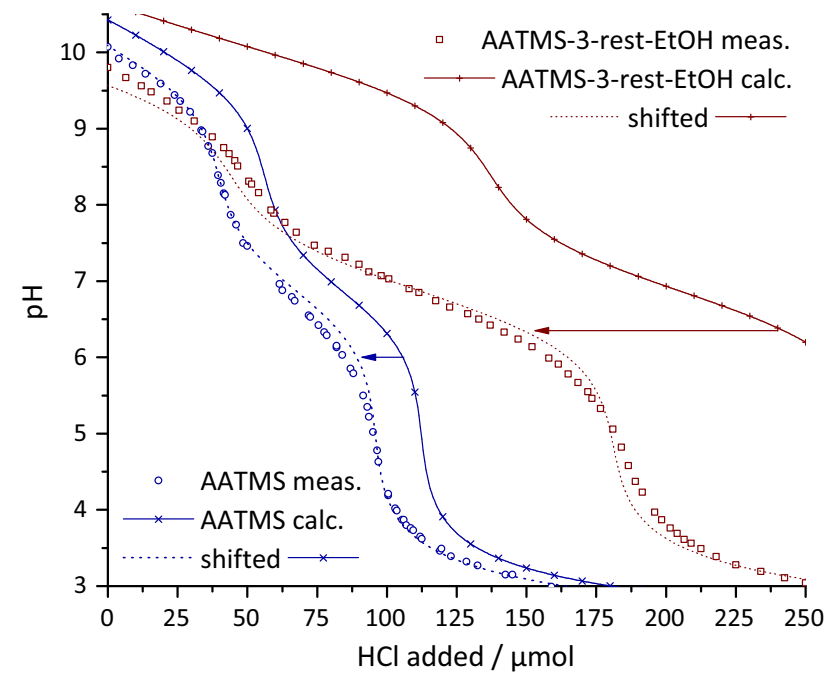

Fig. 3 Measured and calculated titration curves of AATMS and AATMS-3-rest-EtOH possibly resulting in $\mathrm{SO}_{x}$ surface groups as well. Considering the titration curves, $0.63 \mathrm{mmol}$ acid groups were present before or created during the AATMS-3 functionalization process. The titration indicates that $1.1 \mathrm{mmol}$ AATMS was left in the used EtOH, which corresponds to $74 \%$ of the amount added initially. Assuming that the residual $26 \%$ of the AATMS molecules adhered to the IF$\mathrm{WS}_{2}$ particles, one can estimate the maximum organic mass fraction of AATMS-3 to $7.2 \%$.

In the case of AATMS-1-rest-EtOH, the $\mathrm{pH}$ is acidic right from the beginning; the titration was therefore done with $10 \mathrm{mmol} / \mathrm{l} \mathrm{NaOH}$ instead. The well-reproducible curve shape is similar to those reported above, as there are once again two $\mathrm{pH}$ steps at the same $\mathrm{pH}$ values (data given in the Online Resource). This indicates that AATMS modifier was present in AATMS-1-rest-EtOH, but more acid groups had been present before or created during the treatment than base groups had been added in the form of AATMS, namely 0.93 mmol. Hence, AATMS-1 is functionalized with diammonium molecules rather than with diamines. The amount of diammonium measured in the sample corresponds to $47 \%$ of the nominally added AATMS. Assuming that the other $53 \%$ adhered on the IF$\mathrm{WS}_{2}$ particles, one can estimate the maximum organic mass fraction of AATMS- 1 to $5.8 \%$.

Comparable titrations were done on the modified powders AATMS-1 and AATMS-3 as well (data given in the Online Resource). AATMS-3 results in a basic $\mathrm{pH}$ value when dispersed within $\mathrm{H}_{2} \mathrm{O}$ while AATMS-1 results in an acidic $\mathrm{pH}$ value, which is in good agreement with the results of the respective EtOH rests. However, the obtained titration curves were not as well defined as those and the measured diamine contents far exceeded the expected amounts. This is most likely due to the participation of the IF-WS ${ }_{2}$ in the acid-base reaction during the titration, e.g., with their oxidized S groups, thereby wrongly indicating very high modifier contents. Quantitative titration results must therefore be interpreted cautiously.

\section{TGA}

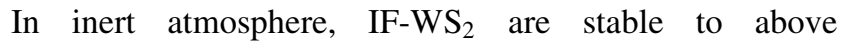
$1000{ }^{\circ} \mathrm{C}$ [10], while organic molecules decompose between 200 and $600{ }^{\circ} \mathrm{C}$; it was therefore expected that the organic content of the treated powders could be measured by TGA. Figure 4 shows the mass of all treated IF-WS 2 powders over temperature. The largest mass loss occurs at $600{ }^{\circ} \mathrm{C}$, when the flow gas is switched to $\mathrm{O}_{2}$; here, the $\mathrm{W}$ of the outermost $\mathrm{WS}_{2}$ layers oxidizes to $\mathrm{WO}_{3}$ and the $\mathrm{S}$ oxidizes, e.g., to $\mathrm{SO}_{2}$, and vaporizes. The yellow color of the residual powder after the measurement indicates $\mathrm{WO}_{3}$ as well.

Even though the powders had been stored in a vacuum exsiccator and dried in a vacuum oven just before the 
measurement, all treated powders, whether functionalized or not, lost $0.8-1.5 \%$ of their mass below $220^{\circ} \mathrm{C}$. This is most likely due to firmly bound $\mathrm{H}_{2} \mathrm{O}$ molecules that are either hard to remove or that are taken up quickly after drying. The presence of $\mathrm{H}_{2} \mathrm{O}$ on $\mathrm{IF}-\mathrm{WS}_{2}$ has been reported earlier and was explained with $\mathrm{H}_{2} \mathrm{O}$ molecules in the voids in the center of the IF-WS 2 as well as in defects in the IF$\mathrm{WS}_{2}$ structure [25].

Generally, powders that were treated similarly show similar mass loss behavior, showing that the mass loss is affected by the silane treatment. However, Ref-CHCl shows significant mass loss as well, even though it contains no surface modifier. This shows that the TGA mass loss alone is not sufficient as a measure for the organic content of IF-WS $\mathrm{W}_{2}$. In contrast, these results once again indicate that the used solvent affects the powders, as the TGA curve of $\mathrm{Ref}-\mathrm{CHCl}_{3}$ is very similar that of the HTCS-modified powders, which have been treated within $\mathrm{CHCl}_{3}$ as well.

The AATMS-modified powders are the only ones whose mass loss behavior is significantly different from that of all other powders, including the reference curves. The significant mass loss around $290{ }^{\circ} \mathrm{C}$ indicates the decomposition of the supposed ammonium in the AATMS molecule, which might work similar to a Hofmann elimination. Thermal loss of ammonium modifiers has been reported earlier to take place at roughly that temperature [26]. This again indicates that the functionalization with AAMTS was successful.

Interestingly, the TGA mass loss correlates strongly with the $\mathrm{C}, \mathrm{O}, \mathrm{N}$, and $\mathrm{SO}_{x}$ contents measured by XPS (see Table 2). This is in good agreement with the assumption that the TGA mass loss is mainly due to removal of $\mathrm{CO}_{2}$, $\mathrm{H}_{2} \mathrm{O}, \mathrm{SO}_{\mathrm{x}}$ and ammonium compounds. Note that the TGA curves of the HTCS-modified powders are entirely different if the tempering step is omitted as unreacted

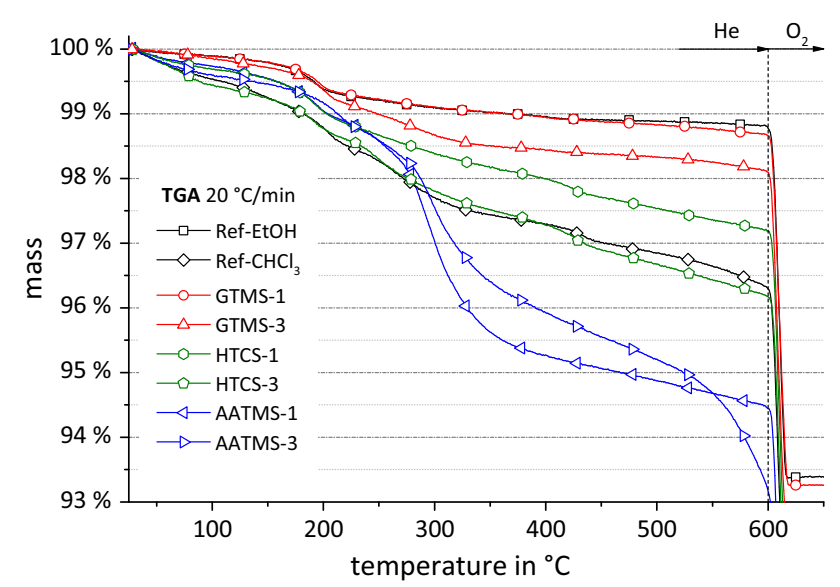

Fig. 4 TGA curves of all treated powders chlorosilanes might then react during the measurement, releasing $\mathrm{HCl}$ (details given in the Online Resource).

\section{TG/MS}

The composition of the gas produced during a TGA measurement was analyzed via TG/MS measurements on AATMS-3 and HTCS-3 in order to obtain additional information on the origin of the mass loss; the analyzed ratios of ion mass $m$ to ion charge $q$ were chosen in a way to analyze possible traces of $\mathrm{CO}_{2}, \mathrm{H}_{2} \mathrm{O}, \mathrm{HCl}$ and ammonium compounds in the gas.

The results support the assumption that the mass loss is mainly due to $\mathrm{H}_{2} \mathrm{O}$ and $\mathrm{CO}_{2}$, and in the case of AATMS-3 possibly also due to succession of ammonium compounds (detailed results and discussion given in the Online Resource). No traces of $\mathrm{HCl}$ were detected, indicating that no unreacted chlorosilanes were left after tempering at $100{ }^{\circ} \mathrm{C}$. The detected $\mathrm{CO}_{2}$ indicates that organic species were present on both samples and hence that these powders had been functionalized successfully. The detection of ammonium compounds further supports this assumption for AATMS-3.

\section{Sonication of untreated IF-WS $\mathbf{W}_{2}$ powders}

During sonication, agglomerates are split into two or more differently large parts until a final agglomerate size is reached. As agglomerates can also reform during sonication, it is possible that not all agglomerates will be split even after infinitely long sonication times, hence the final average number of primary particles per agglomerate $n_{\text {end }}$ might be more than unity. It is assumed here that the reduction of the average number of particles per agglomerate $n$ over time $t$ is proportional to the difference between $n$ and $n_{\text {end }}$ :

$\tau \cdot \frac{\mathrm{d} n}{\mathrm{~d} t}=-\left[n(t)-n_{\mathrm{end}}\right]$

with the proportionality factor $\tau$ being called time constant. Integrating gives:

$n(t)=n_{\mathrm{end}}+\left(n_{0}-n_{\mathrm{end}}\right) \cdot e^{-\frac{t}{\tau}}$

with $n_{0}=n(t=0)$. Due to $n_{0} \gg n_{\text {end }}$ this can be rewritten to:

$n(t)=n_{\mathrm{end}}+n_{0} \cdot e^{-\frac{t}{\tau}}$.

The time $t_{\alpha}$ until a certain ratio $\alpha$ of the final dispersion quality is reached can be calculated:

$1-\alpha=\frac{n\left(t_{\alpha}\right)-n_{\mathrm{end}}}{n_{0}-n_{\mathrm{end}}} \approx \frac{n\left(t_{\alpha}\right)-n_{\mathrm{end}}}{n_{0}}=e^{-\frac{t_{\alpha}}{\tau}}$ 
$t_{\alpha}=-\tau \cdot \ln (1-\alpha)$.

The $n$ in Eq. (3) can be substituted by the average agglomerate volume $V$ or the average agglomerate diameter $d$ due to $n \propto V \propto d^{3}$ :

$\mathrm{d}^{3}(t)=\mathrm{d}_{\text {end }}^{3}+\mathrm{d}_{0}^{3} \cdot e^{\frac{t}{t}}$

In order to measure agglomerate sizes after sonication, SEM imaging was used in a first attempt as it was done in an earlier work [2]. Some differences are visible between SEM images of samples sonicated for very short and very long time periods, respectively (e.g., for $10 \mathrm{~s}$ and $60 \mathrm{~min}$; see Fig. 5, additional images in the Online Resource): while individual primary particles can be seen in most samples, agglomerates of micrometer dimensions are visible more often in samples that had been sonicated for shorter time periods, which shows that sonication is generally able to break agglomerates. However, nanoparticles tend to reagglomerate upon drying due to their van-derWaals interaction, which is the stronger the smaller the particles are. The IF-WS 2 agglomerates had hence quite likely been significantly smaller within the EtOH suspension before it dried. Moreover, SEM images are very difficult to quantify sensibly. In contrast, DLS is done directly on suspensions and is statistically much more useful, which is why it was considered a superior tool for measuring agglomerate size distributions.

The IF-WS ${ }_{2}$ suspension became dark black within a few seconds of sonication, indicating successful dispersion. Nevertheless, large agglomerates were visible as well, even after $60 \mathrm{~min}$ of sonication. Hence, it seems that the sonication resulted in a polydisperse, bimodal agglomerate size distribution. DLS is very sensitive to polydispersity; already few large agglomerates will result in a measurement outlier. Such outliers appeared very frequently, even though most large agglomerates sedimented quickly and thus did not affect the DLS measurement. Unfortunately, the automatic outlier detection by the DLS software did not work reliably enough, which is why some outliers had to be excluded manually. Figure 6 shows the measured $\bar{x}_{\text {DLS }}$ after two different sonication set-ups (different probe diameter, with and without stirring). The empty points indicate excluded data. The remaining data were fitted to Eq. (6) and the fit is given in Fig. 6 with its $95 \%$ confidence interval.

Each sample was measured twice with the DLS, and the results normally agreed well. This shows that the repeatability of the DLS was reasonable, but that some of the samples contained large agglomerates that caused problems. Interestingly, the results appeared to be better when a probe of smaller diameter was used and when the liquid was not stirred. However, this is probably just because the large agglomerates were mostly sedimented on the bottom
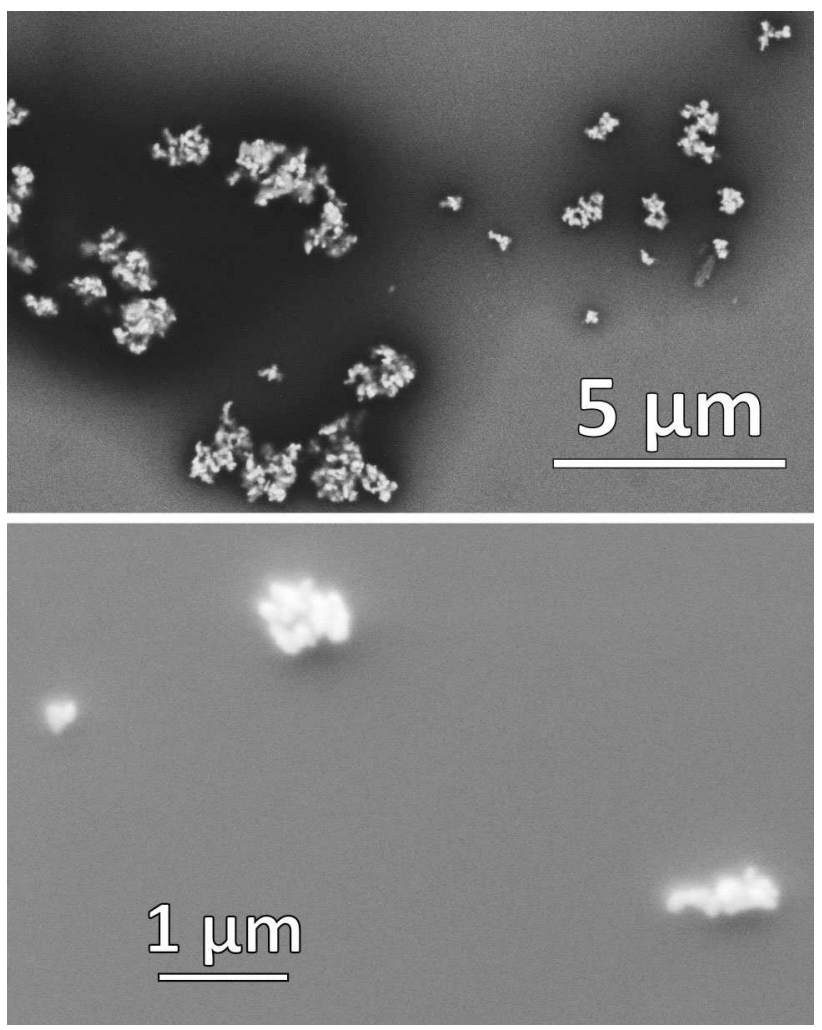

Fig. 5 SEM images of dried Ref-EtOH after sonication for $10 \mathrm{~s}$ (top) and $60 \mathrm{~min}$ (bottom), respectively, showing IF-WS 2 agglomerates

of the beaker and hence fewer of them were collected for measurement. These results are thus probably not representative for the real agglomerate size distribution.

Figure 6 shows that Eq. (6) fits the selected data points quite well and seems to be more appropriate than models suggested elsewhere [14]. The fit parameters are listed in

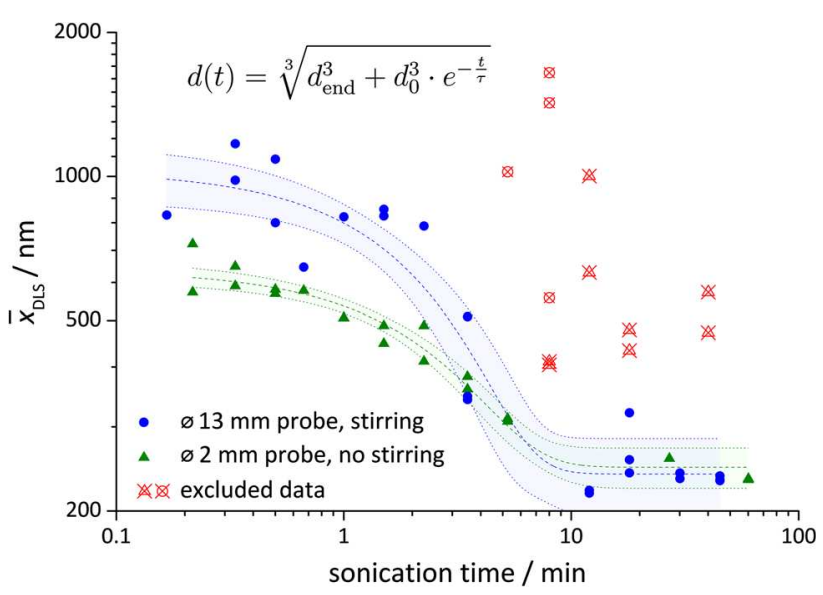

Fig. $6 \bar{x}_{\text {DLS }}$ over sonication time of $\mathrm{IF}_{-\mathrm{WS}_{2}}$ within EtOH as measured by DLS with two different sonication set-ups. The data were fitted using Eq. (6); the bands represent the $95 \%$ confidence interval 
Table 3 Fit parameters of the curves in Fig. 6, giving the final average diameter of particles after sonication $d_{\text {end }}$, their initial diameter $d_{0}$ and the time constant $\tau$ as modeled with Eq. (6)

\begin{tabular}{llrl}
\hline Probe $\varnothing$ & $d_{\text {end }} / \mathrm{nm}$ & \multicolumn{1}{c}{$d_{0} / \mathrm{nm}$} & \multicolumn{1}{l}{$\tau / \mathrm{min}$} \\
\hline $13 \mathrm{~mm}$ & $239 \pm 21$ & $1020 \pm 67$ & $1.29 \pm 0.22$ \\
$2 \mathrm{~mm}$ & $247 \pm 11$ & $626 \pm 16$ & $1.75 \pm 0.18$ \\
\hline
\end{tabular}

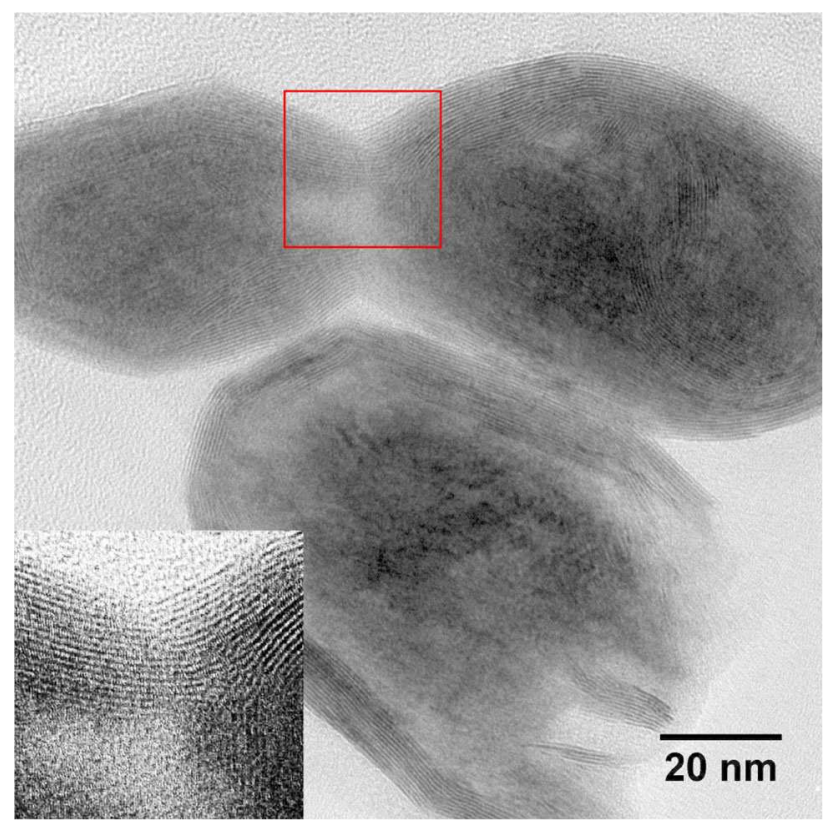

Fig. 7 TEM image of IF-WS 2 nanoparticles after $60 \mathrm{~min}$ of sonication in $\mathrm{EtOH}$. The framed area is magnified in the bottom left corner with enhanced contrast

Table 3: Independently of the set-up, $\tau$ was below $2 \mathrm{~min}$. Inserting these values into Eq. (5) gives $t_{0.99}<10 \mathrm{~min}$, meaning that $99 \%$ of the final dispersion quality will be obtained within $10 \mathrm{~min}$ for both investigated sonication set-ups. The $d_{\text {end }}$ agrees very well for both sonication setups, but in both cases it is still much larger than the diameter of the primary particles. Thus, it seems that agglomerates remain even after long sonication times.

The agglomerate sizes visible in TEM images agree reasonably well with the SEM and DLS results: while individual primary particles do exist, there are also agglomerates of different sizes even after $60 \mathrm{~min}$ of sonication. It seems as if some of the particles were broken up; however, it cannot be stated if these particles had broken up during sonication or if they were broken up already before. Likewise, it is unclear to what extent the nanoparticles had reagglomerated upon drying on the TEM grid, analogous to the possible reagglomeration on the $\mathrm{Si}$ wafers as observed via SEM.

Two of the primary IF-WS ${ }_{2}$ particles in Fig. 7 share some $\mathrm{WS}_{2}$ layers with each other. Hence, the adhesion between them is not just due to van-der-Waals forces but they are conjoined by covalent bonds. Such conjoined nanoparticles could be observed several times. Thus, it seems as if some agglomerates are rather aggregates, or agglomerates of aggregates. The fact that the final agglomerate diameter is significantly larger than that of the primary particles supports the assumption that a significant fraction of the IF-WS ${ }_{2}$ is present in the form of aggregates.

\section{Sonication of treated IF-WS 2 powders}

The treated powders were sonicated within EtOH the same way as the untreated ones (examples given in Fig. 8). Once again, some measurement results had to be excluded arbitrarily, but the data generally obey Eq. (6). The fitting parameters are given in Table 4.

The reference powders and the nominally GTMS-functionalized powders show strongly reduced $d_{\text {end }}, d_{0}$, and with one exception, $\tau$. Thus, the treatment improved the initial dispersion quality as well as the dispersibility of the powders, even though there was no modifier added to the reference powders and the GTMS functionalization was probably unsuccessful. The improvements are most likely due to the short sonication and the grinding with mortar and pestle during the treatment.

In contrast, the successfully functionalized powders had an increased $d_{0}$ and with one exception an increased $d_{\text {end }}$ when compared to the untreated powders; moreover, the scatter was strongly increased. Hence, the silane functionalization deteriorated the dispersibility of IF-WS rather than to improve it, even though the treatment itself was shown to improve the dispersion quality significantly. It seems, therefore, that the silane modifiers caused the IF$\mathrm{WS}_{2}$ agglomerates to stick together.

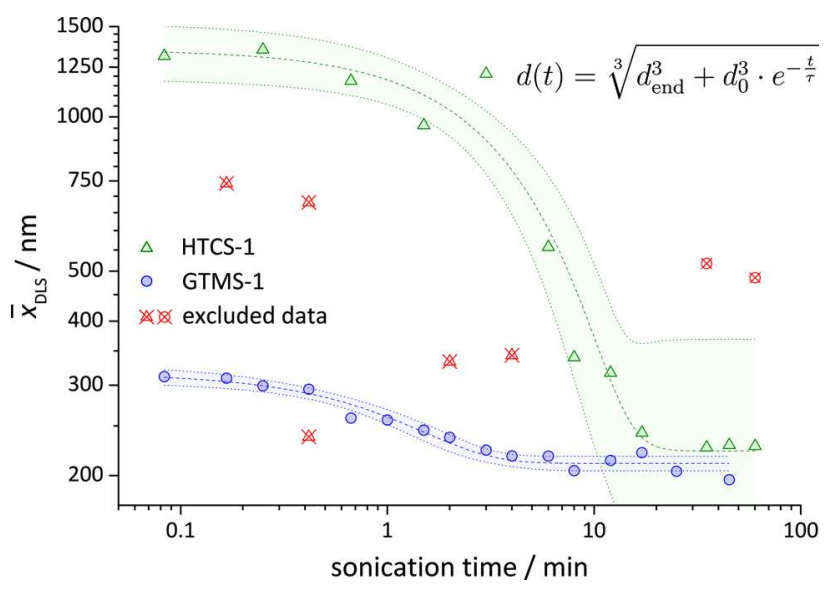

Fig. $8 \bar{x}_{\text {DLS }}$ over sonication time of two treated IF-WS ${ }_{2}$ within EtOH as measured by DLS. The data were fitted using Eq. (6); the bands represent the $95 \%$ confidence interval 
Table 4 Fit parameters of the DLS measurement results of all treated $\mathrm{IF}_{-} \mathrm{WS}_{2}$ dispersed within EtOH, fitted by Eq. (6)

\begin{tabular}{lccl}
\hline & $d_{\text {end }} / \mathrm{nm}$ & $d_{0} / \mathrm{nm}$ & $\tau / \mathrm{min}$ \\
\hline Ref-EtOH & $210 \pm 4$ & $306 \pm 12$ & $0.73 \pm 13$ \\
Ref-CHCl $_{3}$ & $228 \pm 11$ & $384 \pm 27$ & $0.70 \pm 24$ \\
GTMS-1 & $211 \pm 4$ & $282 \pm 8$ & $0.99 \pm 15$ \\
GTMS-3 & $222 \pm 3$ & $345 \pm 7$ & $1.85 \pm 22$ \\
AATMS-1 & $422 \pm 34$ & $1226 \pm 145$ & $1.76 \pm 23$ \\
AATMS-3 & $620 \pm 52$ & $1714 \pm 204$ & $1.41 \pm 14$ \\
HTCS-1 & $223 \pm 64$ & $1350 \pm 75$ & $2.43 \pm 45$ \\
HTCS-3 & $309 \pm 36$ & $1844 \pm 440$ & $0.11 \pm 04$ \\
\hline
\end{tabular}

These results indicate that surface functionalization of IF-WS ${ }_{2}$ with organic molecules is possible, but does not necessarily improve its dispersibility in fairly polar solvents like EtOH. The forces holding the individual IF-WS particles together are very small to begin with and are hence not reduced by silane surface modifiers. Possible positive effects of the surface functionalization on the longterm dispersion stability [2] might not be related to improved dispersion quality. Likewise, the enhanced proper-

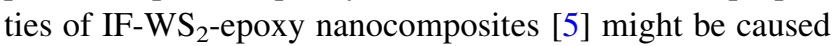
by other factors.

\section{Conclusion and outlook}

This work deals with three types of silane surface functionalization of IF-WS $\mathrm{W}_{2}$ their characterization and their dispersion within EtOH. Various measurements indicate that the modifications with HTCS and AATMS were successful while that with GTMS was not. The most direct evidence for successful modification was the presence of the $\mathrm{Si}_{2 p}$ peak in the XPS curves, while the increase in the $\mathrm{C}, \mathrm{O}$, or $\mathrm{N}$ contents were considered inconclusive.

The performed treatment, both with and without silane modifiers added, resulted in the formation of $\mathrm{SO}_{x}$ groups, most likely $\mathrm{SO}_{2}$ or $\mathrm{SO}_{3}$. These decrease the $\mathrm{pH}$ of the suspension and can hence partially or fully protonate the surface modifier. As long as this effect is taken into account, acid-base titration of the residual solvent left from the treatment can be used to determine indirectly the presence and to some extent the quantity of basic or acidic surface modifier on the nanoparticles.

Strongly bound $\mathrm{H}_{2} \mathrm{O}$ was still present on the IF-WS powders after drying them at $50{ }^{\circ} \mathrm{C}$ in vacuum for several hours. Together with the evaporation of $\mathrm{SO}_{x}$, this complicates the determination of organic contents with TGA. Only the amine-functionalized IF-WS 2 could be clearly discriminated from the references.
The powders were dispersed within EtOH by sonication down to agglomerate diameters of approx. $200 \mathrm{~nm}$, which is still larger than the nominal average primary particle size (around $100 \mathrm{~nm}$ ). The development of agglomerate sizes over sonication time could be measured by DLS reasonably well, although several outliers had to be excluded, likely caused by bimodal polydispersity. Equation (6) approximated the evolution of the measured agglomerate size over sonication time reasonably well. After $10 \mathrm{~min}$, further sonication does not seem to reduce the agglomerate sizes any further. TEM images show that some of the primary particles were conjoined with each other, forming aggregates; this might explain why the agglomerate size could not be reduced any further.

The performed treatment can hence help to limit the initial agglomerate size and to accelerate the size reduction upon sonication, but barely reduces the final agglomerate size. Moreover, the successfully functionalized powders showed significantly inferior dispersibility in $\mathrm{EtOH}$ to the unfunctionalized references. This shows that the chemical interaction between the IF-WS $\mathrm{W}_{2}$ and the dispersion solvent is not the factor limiting the dispersion quality.

In an upcoming work, these IF-WS 2 powders will be used to manufacture epoxy nanocomposites with special focus on the resulting fracture toughness. Special emphasis will be given to the obtained dispersion quality and the bonding between the IF-WS $\mathrm{W}_{2}$ particles and the epoxy matrix.

Acknowledgements The authors would like to thank Anja Huch, Beatrice Fischer, Jörg Patscheider, Lassi Karvonen and Matthias Nagel for their experimental and theoretical support.

\section{References}

1. Rapoport L, Bilik Y, Feldman Y, Homyonfer M, Cohen SR, Tenne R (1997) Hollow nanoparticles of $\mathrm{WS}_{2}$ as potential solidstate lubricants. Nature 387:791-793. doi:10.1038/42910

2. Shahar C, Zbaida D, Rapoport L, Cohen H, Bendikov T, Tannous J, Dassenoy F, Tenne R (2010) Surface functionalization of $\mathrm{WS}_{2}$ fullerene-like nanoparticles. Langmuir 26(6):4409-4414. doi:10. 1021/la903459t

3. Naffakh M, Díez-Pascual AM, Marco C, Ellis GJ, Gómez-Fatou MA (2013) Opportunities and challenges in the use of inorganic fullerene-like nanoparticles to produce advanced polymer nanocomposites. Prog Polym Sci 38(8):1163-1231. doi:10.1016/ j.progpolymsci.2013.04.001

4. Buchman A, Dodiuk-Kenig H, Dotan A, Tenne R, Kenig S (2009) Toughening of epoxy adhesives by nanoparticles. J Adhes Sci Technol 23(5):753-768. doi:10.1163/156856108X379209

5. Shneider M, Dodiuk H, Tenne R, Kenig S (2013a) Nanoinduced morphology and enhanced properties of epoxy containing tungsten disulfide nanoparticles. Polym Eng Sci 53(12):2624-2632. doi:10.1002/pen.23517

6. Ghoreishi SM, Meshkat SS, Ghiaci M, Dadkhah AA (2012) Nanoparticles synthesis of tungsten disulfide via AOT-based 
microemulsions. Mater Res Bull 47(6):1438-1441. doi:10.1016/j. materresbull.2012.02.042

7. Shneider M, Rapoport L, Moshkovich A, Dodiuk H, Kenig S, Tenne R, Zak A (2013b) Tribological performance of the epoxybased composite reinforced by $\mathrm{WS}_{2}$ fullerene-like nanoparticles and nanotubes. Phys Status Solidi A 210(11):2298-2306. doi:10. 1002/pssa.201329162

8. Xu F, Yan C, Shyng YT, Chang H, Xia Y, Zhu Y (2014) Ultratoughened nylon 12 nanocomposites reinforced with IF-WS Nanotechnology 25(32):325701. doi:10.1088/0957-4484/25/32/ 325701

9. Späth B, Kopnov F, Cohen H, Zak A, Moshkovich A, Rapoport L, Jägermann W, Tenne R (2008) X-ray photoelectron spectroscopy and tribology studies of annealed fullerene-like $\mathrm{WS}_{2}$ nanoparticles. Phys Status Solidi B 245(9):1779-1784. doi:10. 1002/pssb.200779531

10. NanoMaterials Ltd. (2011) Material safety data sheet NanoLub R, $\mathrm{WS}_{2}$ lubricant nanopowder

11. ISO 3696:1987 (1987) Water for analytical laboratory usespecification and test methods. Standard, International Organization for Standardization, Geneva

12. Gutz IGR (version 4) CurTiPot-pH and acid-base titration curves: analysis and simulation freeware

13. Merkus HG (2009) Particle size measurements, Chap 5. Dispersion of powders in air and in liquids. Springer, New York, pp 117-136. doi:10.1007/978-1-4020-9016-5

14. Bittmann B, Haupert F, Schlarb AK (2009) Ultrasonic dispersion of inorganic nanoparticles in epoxy resin. Ultrason Sonochem 16(5):622-628. doi:10.1016/j.ultsonch.2009.01.006

15. Bittmann B, Haupert F, Schlarb AK (2011) Preparation of $\mathrm{TiO}_{2} /$ epoxy nanocomposites by ultrasonic dispersion and their structure property relationship. Ultrason Sonochem 18(1):120-126. doi:10.1016/j.ultsonch.2010.03.011

16. Bittmann B, Haupert F, Schlarb AK (2012) Preparation of $\mathrm{TiO}_{2}$ epoxy nanocomposites by ultrasonic dispersion and resulting properties. J Appl Polym Sci 124(3):1906-1911. doi:10.1002/ app.34493
17. Englert M, Bittmann B, Haupert F, Schlarb A (2012a) Scaling-up of the dispersion process of nanoparticle-agglomerates in epoxy resin with an innovative continuous ultrasonic flow-through-cell dispersion system. Polym Eng Sci 52(1):102-107. doi:10.1002/ pen.22051

18. Englert M, Haupert F, Schlarb AK (2012b) Verification of a dispersion model to describe the dispersion of nanoparticle agglomerates in epoxy resin with a stirred bead mill. J Dispersion Sci Technol 33(8):1240-1246. doi:10.1080/01932691.2010. 505112

19. ISO 22412:2008(E) (2008) Particle size analysis—dynamic light scattering (DLS). Standard, International Organization for Standardization, Geneva

20. Alexander MR, Short RD, Jones FR, Stollenwerk M, Zabold J, Michaeli W (1996) An X-ray photoelectron spectroscopic investigation into the chemical structure of deposits formed from hexamethyldisiloxane/ oxygen plasmas. J Mater Sci 31(7):18791885. doi:10.1007/BF00372203

21. Flint EB, Suslick KS (1991) The temperature of cavitation. Science 253(5026):1397-1399. doi:10.1126/science.253.5026.1397

22. Haynes WM (2014) Dissociation constants of organic acids and bases. CRC handbook of chemistry and physics, 95th edn. CRC Press, Boca Raton, pp 5-94

23. Feldman Y, Zak A, Popovitz-Biro R, Tenne R (2000) New reactor for production of tungsten disulfide hollow onion-like (inorganic fullerene-like) nanoparticles. Solid State Sci 2(6): 663-672. doi:10.1016/S1293-2558(00)01070-0

24. Tenne R, Seifert G (2009) Recent progress in the study of inorganic nanotubes and fullerene-like structures. Annu Rev Mater Res 39(1):387-413. doi:10.1146/annurev-matsci-082908-145429

25. Panich AM, Kopnov F, Tenne R (2006) Nuclear magnetic resonance study of fullerene-like $\mathrm{WS}_{2}$. J Nanosci Nanotechnol 6(6):1678-1683. doi:10.1166/jnn.2006.211

26. Chattopadhyay D, Galeska I, Papadimitrakopoulos F (2003) A route for bulk separation of semiconducting from metallic singlewall carbon nanotubes. J Am Chem Soc 125(11):3370-3375. doi:10.1021/ja0285991 